\title{
5 Weitere therapeutische Maßnahmen und Prophylaxe
}

\subsection{Hautschutz}

\subsubsection{Wundumgebung und Störfaktoren der Hautbarriere}

Die Wundumgebung ist die an den Wundrand oder die Wundfläche reichende original geschichtete Haut (Definition DGfW).

Eine intakte Haut ist der beste Hautschutz. Durch folgende Störfaktoren kann die Hautbarriere funktionell eingeschränkt werden:

- Durch Einwirkung des Wundexsudates in der Wundumgebung kommt es zu Mazeration und nachfolgend zu Hautentzündungen (kumulativ-toxisches Kontaktekzem) mit Ausbildung von Erosionen/Ulzerationen und damit Vergrößerung der ursprünglichen Wunde.

- Durch Einwirkung von Hautpflegeprodukten und Wundtherapeutika können allergische oder toxische Kontaktekzeme entstehen, die die Wundheilung behindern.

- Bei Patienten mit chronischen Wunden ist die Gefahr von Kontaktallergien gegenüber Duftstoffen, Salbenbestandteilen oder anderen Inhalten von Lokaltherapeutika deutlich erhöht.

- Durch systemische Grunderkrankungen kann die Hautbarriere maßgeblich geschädigt sein:

○ Diabetes mellitus: trockene anhydrotische Haut mit veränderter Immunkompetenz und damit veränderter Hautschutzbarriere.

- Chronisch venöse Insuffizienz: Ödeme der Dermis führen zu sekundären Stauungsekzemen und nachfolgend zu Juckreiz, sekundärem Lymphödem etc. mit Abheilungsverzögerung von Wunden.

- Periphere arterielle Verschlusskrankheit (pAVK): aufgrund der Minderdurchblutung verminderte Hautbarrierefunktion.

- Es gibt zahlreiche weitere Erkrankungen, die die Hautbarrierefunktion verändern, wie Lymphödeme, Kollagenosen etc.

Die Aufklärung von Patient und Angehörigen ist maßgeblich für eine erfolgreiche Prävention von Hautschädigungen. 


\subsubsection{Pflege der Wundumgebung}

Der Schutz der umgebenden Haut ist wesentlicher Bestandteil der Behandlung chronischer Wunden.

Bei der Hautreinigung auch durch sogenannte $\mathrm{pH}$-neutrale Seifen wird der Säureschutzmantel angegriffen oder sogar vollständig zerstört. Dies ist abhängig vom jeweiligen Zustand der Haut, der Häufigkeit der Reinigungsmaßnahmen und der Effektivität nachfolgender rehydrierender Pflege.

Oft ist ein Waschen mit körperwarmem Wasser ohne Seifenzusätze vollkommen ausreichend. Eine trockene Haut ist anschließend mit Produkten auf Wasser-in-Öl-Basis zu pflegen. Die Anwendung solcher Produkte spendet Feuchtigkeit und hält die Haut durch einen feinen Fett-Wasser-Film weich und geschmeidig.

Produkte auf Basis von Öl-in-Wasser sollten bei problematischer Haut nicht zum Einsatz kommen. Der sogenannte Dochteffekt entzieht der Haut mehr Feuchtigkeit, als die Lotion mitbringt.

Gele sind halbfeste, wässrige Zubereitungen. Es handelt sich meist um Mischungen aus Wasser, Glyzerol oder Propylenglykol. Sie sind durchsichtig, fettfrei, wirken kühlend durch den hohen Wasseranteil, haben jedoch oft keinen hautpflegenden Effekt.

Öle, Fette und Salben decken die Hautporen häufig regelrecht ab und behindern dadurch den Gasaustausch der Haut. Erschwerend kommt hinzu, dass die Wundauflagen nicht mehr haften können.

Reine Fettpräparate sollten in der Regel nicht zur Hautpflege eingesetzt werden. Besonders Produkte aus Erdölraffinaten wie Paraffin und Vaseline schließen die Haut luftdicht ab, verstopfen die Poren und führen so zu einem Hitzestau.

Nicht allgemein bekannt ist, dass Melkfett im Wesentlichen nichts anderes ist als Vaseline und sich für die Hautpflege wenig eignet. Sinnvoll ist der Einsatz nur bei rissiger Hornhaut, z. B. an den Füßen.

Der natürliche Feuchthaltefaktor der Haut (NMF) bindet Wasser an sich und hält die Feuchtigkeit in der Hornschicht fest. Ergänzend können zur Hydratation harnstoffhaltige Präparate etc. zur Hautpflege eingesetzt werden. Weitere Feuchthaltesubstanzen, die in Pflegeprodukten verwendet werden, sind den Bestandteilen des natürlichen Faktors sehr ähnlich.

Der unmittelbare Hautschutz kann mit weicher Zinkpaste erfolgen, dies hat aber den Nachteil, dass eine Hautbeobachtung nicht mehr möglich ist. Dies gilt auch für die Anwendung von Farbstoffen und Pudern, die eine Hautbeobachtung unmöglich machen und die Haut zusätzlich weiter austrocknen. Besser geeignet sind Produkte, die einen hauchdünnen Acrylat-Barrierefilm hinterlassen, der einen Schutz vor Flüssigkeiten/Reizstoffen wie Wundexsudat, Urin, Kot, Fistelsekreten etc. bietet. 
Dieser Hautschutz ist transparent und ermöglicht daher die weitere Beobachtung der Haut. Der Einsatz von abdeckenden Externa, wie Salben etc., ist möglichst zu vermeiden. Bei Vorhandensein von Kontaktekzemen kann der vorübergehende Einsatz von kortikoidhaltigen Externa erforderlich sein. Wichtig ist hierbei der Versuch, den Auslöser zur eruieren.

Bei der Applikation des Hautschutzes sind insbesondere auch intakte Hautareale innerhalb der Wunde, wie Stege oder Inseln, zu beachten. Auch diese müssen geschützt werden.

Zusätzlich sollte der Wundrand (ebenso wie die Wunde selbst) vor Druck und vor Traumata geschützt werden. Dieses erfolgt insbesondere durch eine angemessene Lagerung des betroffenen Körperbereiches.

Die schon geschädigte Umgebungshaut wird häufig zusätzlich noch durch die Klebefixation von Wundauflagen weiter geschädigt. In der Regel sind das Acrylate, die durch ihre hervorragenden Klebeeigenschaften bei wiederholter Anwendung einen sogenannten Strippingeffekt der oberen Epidermis-Schichten bewirken. Daher sind Wundauflagen ohne Klebefläche oder hautfreundliche, mit Silikon beschichtete Klebeauflagen sowie unbeschichtete HydrogelKompressen zu bevorzugen. Dies gilt insbesondere bei Pergament-, Kortisonoder sehr dünner Altershaut.

Der Zustand vorgeschädigter Haut sollte nicht zusätzlich durch Massagen, Lymphdrainagen oder sonstige mechanische Alterationen wie das Einmassieren von Salben weiter verschlechtert werden.

\subsubsection{Hautschutzprodukte}

\section{Hautschutz zur Prophylaxe}

\section{Produkte}

\section{Gelistet in den Helios-Kliniken}

Cavilon Hautschutz (3M) Creme ( $\triangleright$ Abb. 5.1), Spray ( $\triangleright$ Abb. 5.2), Applikator ( Abb. 5.3).

\section{Vergleichbare Produkte}

- Askina Barrier (BBR) Creme, Spray, Applikator

- Cutimed PROTECT (BSN) Creme, Spray, Applikator

- Secura (S\&N) Tücher, Spray, Applikator

- Sesi-Care (CON) Tücher, Spray, Applikator. 


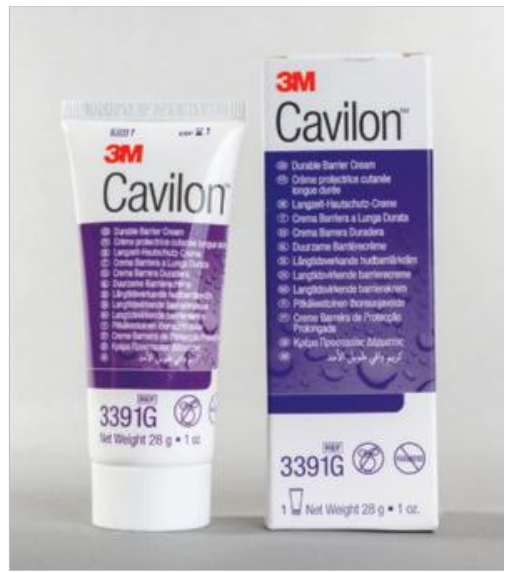

Abb. 5.1 Cavilon Hautschutz: Creme.

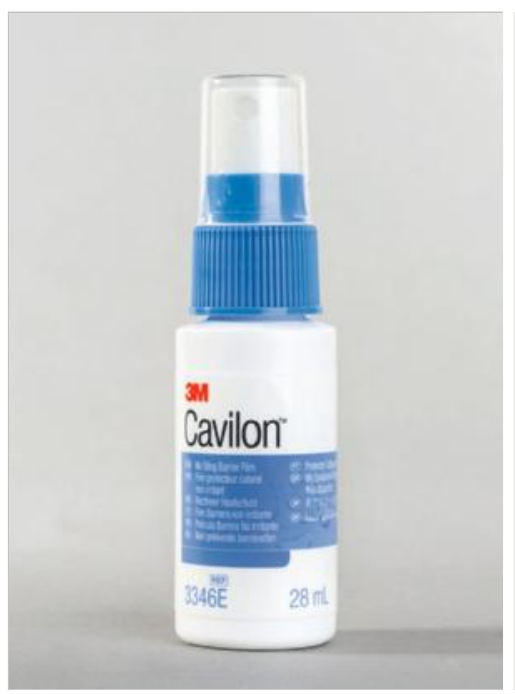

Abb. 5.2 Cavilon Hautschutz: Spray. 


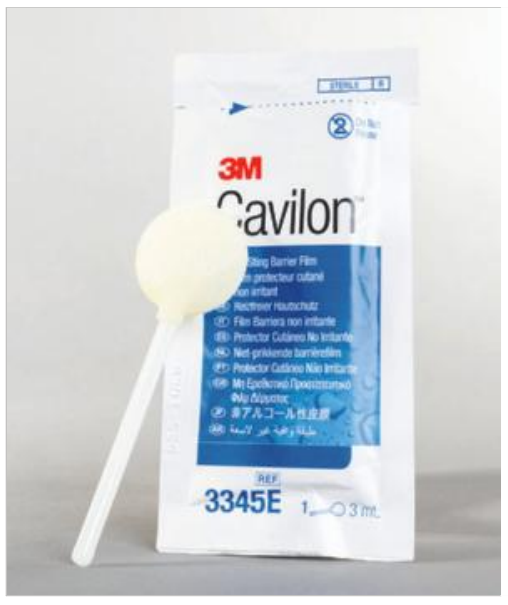

Abb. 5.3 Cavilon Hautschutz: Applikator.

\section{Beschreibung}

Die pH-neutrale, hypoallergene und reichhaltige Creme bietet einen langanhaltenden Hautschutz vor Körperflüssigkeiten und ermöglicht gleichzeitig die Haftung von klebenden Verbänden. Die Creme bildet einen wasserfesten und luftdurchlässigen Hautschutz.

Übersicht s. > Abb. 5.4

\section{Indikation}

Schutz gefährdeter Haut vor austretenden Körperflüssigkeiten und Wundexsudat.

\section{Kontraindikationen}

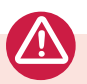

- Cavilon sollte nicht bei infizierter Haut und Hautirritationen angewendet werden.

- Bei sogenannter „Pergamenthaut“ sollte das Produkt mit Vorsicht verwendet werden. 


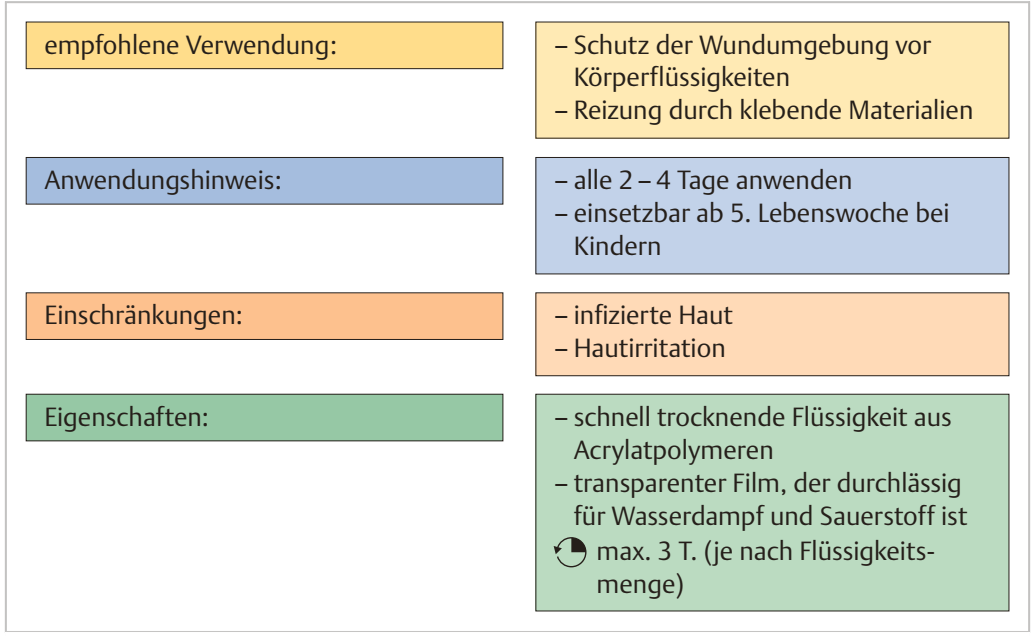

Abb. 5.4 Hautschutz zur Prophylaxe.

\section{Anwendungsweise}

Vor Anwendung sollte die Haut, wenn möglich, gereinigt und trocken sein. Die gleichzeitige Anwendung von Salben, Cremes, Lotionen oder anderen Hautschutzmitteln führt zu Auflösung des Hautschutzfilms.

Die Anwendung bei Kindern ist ab der 5. Lebenswoche möglich.

Verbandwechsel: Das Anwendungsintervall liegt bei 48-72 Stunden (Applikator + Spray), bei häufiger Reinigung oder Verwendung der Creme ist ein erneute Anwendung auch bereits nach 12-24 Stunden notwendig.

\section{Hochleistungsfähiger Hautschutz}

\section{Produkte}

\section{Gelistet in den Helios-Kliniken}

Cavilon Advanced (3M) ( Abb. 5.5). 


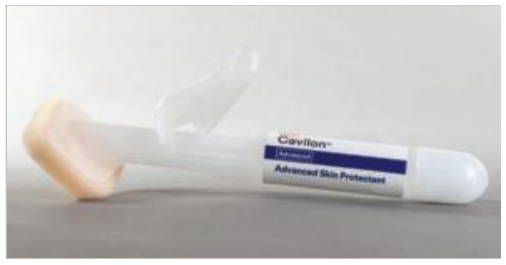

Abb. 5.5 Cavilon Advanced.

\section{Beschreibung}

Cavilon Advanced bildet einen 3-7 Tage anhaltenden, ultradünnen transparenten hochleistungsfähigen Hautschutzfilm, der geeignet ist, empfindliche oder mazerierte/alterierte Haut vor weiteren Schädigungen zu schützen. Er wird mit einem Schwamm-Applikator flüssig aufgebracht und trocknet innerhalb einer Minute zu einem Film aus.

Übersicht s. > Abb. 5.6.

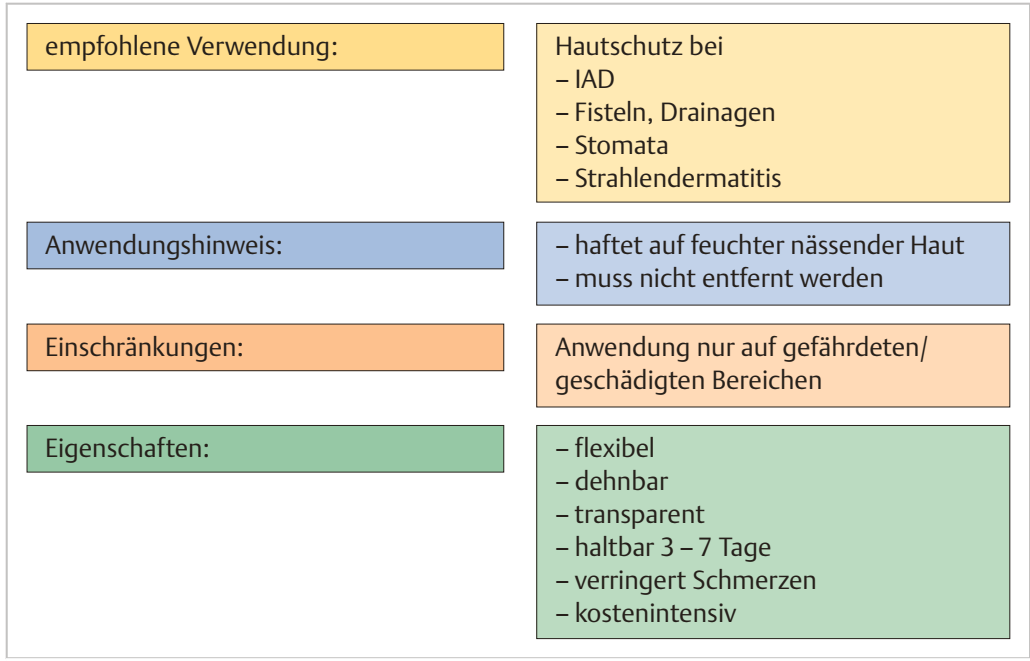

Abb. 5.6 Gesonderter Hautschutz. 


\section{Indikation}

- zum Bedecken und zum Schutz von intakter oder geschädigter Haut, auch vor Körperflüssigkeiten oder Reizstoffen

- beugt Verletzungen der Haut vor und hält sie intakt

- der Barrierefilm lindert sekundär Schmerzen, die mit Inkontinenz-assoziierter Dermatitis (IAD) einhergehen.

\section{Kontraindikationen}

- als Wundverband bei tiefen Wunden

- in den Augen oder im direkten Umfeld.

\section{Cave}

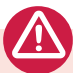

Bei Anwendung von Cavilon Advanced ist die gleichzeitige Verwendung von Reinigungstüchern, Lotionen, Cremes und Pasten, die eine Schutzfunktion enthalten, kontraindiziert.

\section{Anwendungsweise}

- Haut reinigen

- Applikator durch Druck aktivieren, 10 Sekunden warten, bis der Schwamm getränkt ist (nach unten halten, • Abb. 5.7)

- mit Einweg-Applikationsstift auftragen; max. 2-mal in der Woche

- Inhalt: 2,7 ml $\rightarrow$ reicht für die Fläche eines DIN-A4-Blattes

- einzeln verpackt, für den einmaligen Gebrauch vorgesehen

- die Lösung verhindert mikrobielles Wachstum.

Während der Verwendung von Cavilon Advanced kann die Hautreinigung auf 3 Arten erfolgen:

- sanfte Flüssigreiniger mit hautneutralem ph-Wert ohne Schutzbarriere

- ein Wasch- und Reinigungstuch ohne Schutzbarriere verwenden

- die Haut mit klarem Wasser reinigen. 

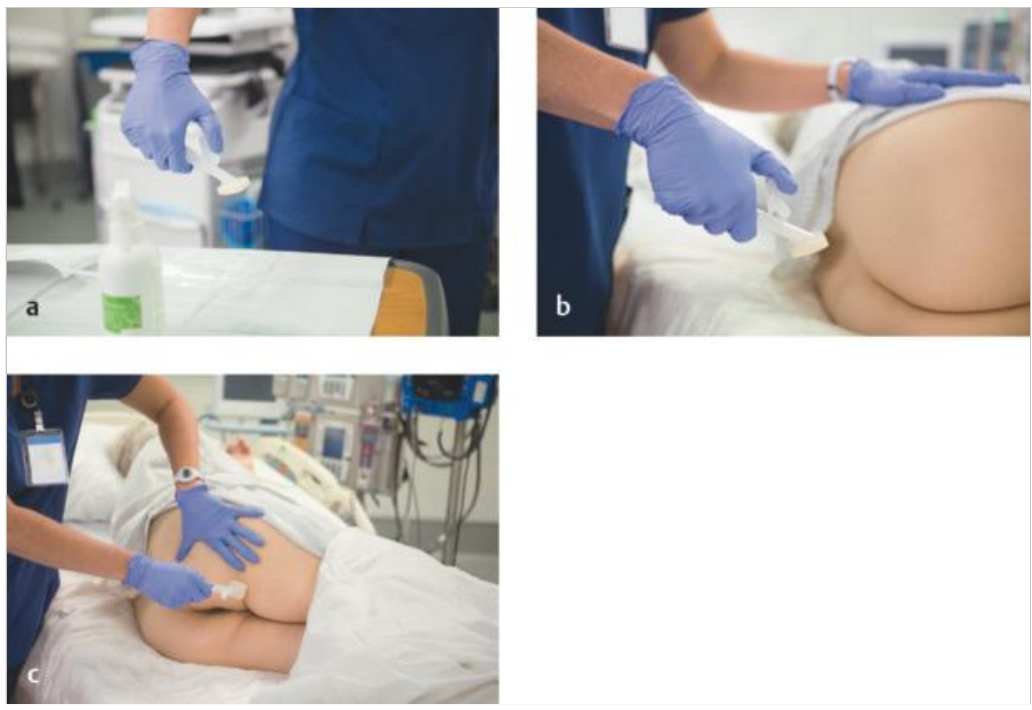

Abb. 5.7 Anwendung des Cavilon-Applikators. (Quelle: Mit freundlicher Genehmigung der 3M Deutschland $\mathrm{GmbH}$ )

a Mit dem Hebel wird durch Zerbrechen der Ampulle das System aktiviert.

b Applikator 10 Sekunden nach unten halten zur Anfeuchtung des Schwammes.

c Gleichmäßiges Auftragen der Flüssigkeit auf der Haut.

\section{Merke}

Trocknungszeit vor Anlegen eines Verbandes: 60 Sekunden.

Sekundärabdeckung: nicht notwendig, nur zum Auffangen von Körperflüssigkeiten.

\section{Einsatz von Cavilon Hautschutz vs. Cavilon Advanced}

Das Grundversorgungsprodukt bei chronischen Wunden ist Cavilon, bei Mazerationsrisiko (z. B. IAD durch Inkontinenz etc.) ist das wesentlich teurere Produkt Cavilon Advanced indiziert ( $\triangleright$ Tab. 5.1). 
Tab. 5.1 Indikation von Cavilon Hautschutz vs. Cavilon Advanced.

\begin{tabular}{|l|l|l|}
\hline & $\begin{array}{l}\text { Cavilon reizfreier } \\
\text { Hautschutz }\end{array}$ & Cavilon Advanced \\
\hline $\begin{array}{l}\text { Prophylaxe im Bereich von Stomata, } \\
\text { Tuben und Fisteln }\end{array}$ & $\times$ & \\
\hline $\begin{array}{l}\text { Wundrandprophylaxe } \\
\text { MARSI-Prophylaxe (Medical-Adhesive- } \\
\text { related Skin Injuries; durch Klebe- } \\
\text { stoffe verursacht) }\end{array}$ & $\times$ & \\
\hline \begin{tabular}{l} 
IAD-Prophylaxe: hohes Risiko \\
\hline $\begin{array}{l}\text { IAD schwere Schädigung } \\
\text { Wundrand: mäßige bis schwere }\end{array}$
\end{tabular} & \\
\hline $\begin{array}{l}\text { Schädigung } \\
\text { Hautschädigung an schwer } \\
\text { zugänglichen Körperstellen }\end{array}$ & & $\times$ \\
\hline $\begin{array}{l}\text { Stomata, Fisteln, und Drainagen: } \\
\text { mäßige bis schwere Schädigung }\end{array}$ & & $\times$ \\
\hline
\end{tabular}

\subsection{Kompressionstherapie}

\section{Merke}

Bei Patienten mit chronischen Wunden mit Beinödemen und beinahe allen Patienten mit Ulcus cruris venosum ist die Kompressionstherapie unerlässlicher Bestandteil der Behandlung.

\subsubsection{Definition}

Der phlebologische Kompressionsverband übt einen definierten Druck aus und erhöht damit den venösen und den lymphatischen Abstrom bei insgesamt verbesserter venöser Pumpfunktion. Es gibt Wechselverbände, die täglich neu angelegt und in der Regel nachts nicht verwendet werden, und Kompressionssysteme mit bis zu 7 Tage Liegedauer. Wir unterscheiden den Unterschenkel bis unterhalb der Knieregion und den Oberschenkel einschließende Verbände oder auch Strumpfsysteme (Konfektion oder nach Maß angefertigt). Es werden vorwiegend längselastische Binden zur Kurzzug- oder auch Langzuganwendung, aber auch nicht dehnbare Verbände wie Zinkleim unterschieden. 


\subsubsection{Indikation}

- venöse Gefäßerkrankungen, wie Varizen (konservativ sowie prä- und/oder postoperativ, ohne/mit Entzündung, auch bei Schwangerschaften), Thrombosen, sowie bei sekundären Folgen wie venös oder lymphatisch bedingten Ödemen und/oder Unterschenkelulzera

- Stauungsbefunde infolge Immobilität (arthrogenes Stauungssyndrom, Dependency-Syndrom, Extremitätenparesen)

- Sonstige Indikationen wie hormonell oder traumatisch bedingte Ödeme etc.

\subsubsection{Anwendung}

\section{Therapieziel}

- Kompression der dilatierten oberflächlichen Beinvenen und des Lymphsystems durch Rückführung von Gewebeflüssigkeit in die Gefäße

- Verbesserung des venösen Rückflusses aus den tiefen Beinvenen durch Erhöhung des Muskeltonus der Wadenmuskelpumpe

- Verhinderung von Thrombosen.

\section{Therapiemöglichkeiten}

- Mehrkomponenten-Kompressionssystem

- Thrombosestrümpfe Konfektion/nach Maß.

\section{Beschreibung}

Mehrkomponenten-Kompressionssysteme bestehen z.B. aus einer weißen polsternden Kurzzugbinde und einer hautfarbenen kohäsiven Langzugbinde die nur komplett angelegt anzuwenden sind. 


\section{Produkte}

\section{Gelistet in den Helios-Kliniken}

- UrgoK2 ( $\triangleright$ Abb. 5.8)

- Kompressionsdruck $40 \mathrm{mmHg}$

- aufgedruckter Kreis als Druckindikator

- UrgoK2 Lite ( $\triangleright$ Abb. 5.9)

- Kompressionsdruck $20 \mathrm{mmHg}$

- eignet sich zum Einstieg in die Kompressionstherapie

- für Patienten, die eine starke Kompression nicht tolerieren

- aufgedrucktes „R“ im Kreis als Druckindikator auf der weißen Binde deutet auf die reduzierte Kompression hin.

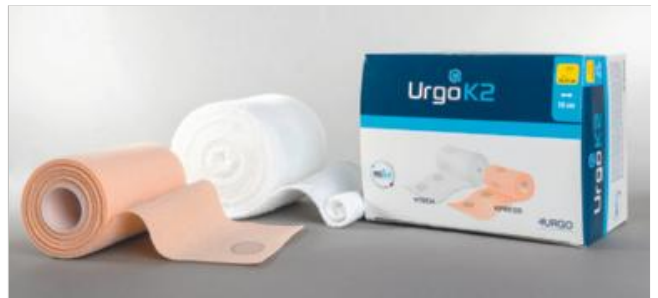

Abb. 5.8 UrgoK2.

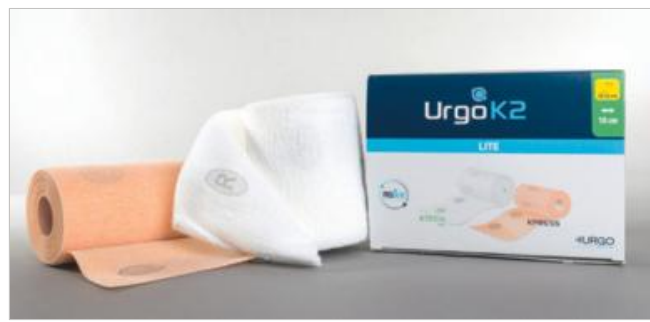

Abb. 5.9 UrgoK2 Lite. 


\section{Durchführung}

- vor Beginn einer Kompressionstherapie Gefäßstatus erheben (Puls tasten und/oder ABI-Messung)

- Verband möglichst am Morgen vor dem ersten Aufstehen oder nach längerer Beinhochlagerung anlegen $\rightarrow$ orthostatische Ödeme reduzieren

- bei Vorliegen einer Wunde zuerst geeigneten Wundverband anbringen

- nach Beinreinigung und Hautpflege erfolgt das Wickeln der Beine im Liegen, am besten in Rückenlage durch geübtes Fachpersonal (Schulungen durch Hersteller möglich)

- je nach Bedarf, Erforderlichkeit und ärztlicher Anordnung wird der Verband bis zum Knie oder bis zu Oberschenkel angelegt

- Form der Gliedmaßen beurteilen $\rightarrow$ Anomalien und potenzielle Druckstellen erkennen

- nach Beendigung der Entstauungsphase und Erreichen der Erhaltungsphase sollte auf individuell angepasste Kompressionsstrümpfe oder -systeme gewechselt werden

- Empfehlung: nach 30 Minuten Durchblutung, Motorik und Sensibilität (DMS) und Sitz des Verbandes kontrollieren.

\section{Merke}

Beachte unterschiedliche Anpressdrücke:

- UrgoK2: ca. $40 \mathrm{mmHg}$

- UrgoK2 Lite: ca. $20 \mathrm{mmHg}$.

$\rightarrow$ Die aufgedruckten Druckindikatoren (Ellipsen im Ruhezustand, > Abb. 5.10) auf beiden Binden erleichtern die korrekte Anlage des Kompressionsverbandes durch Dehnen bis der Druckindikator sich als Kreis darstellt. 

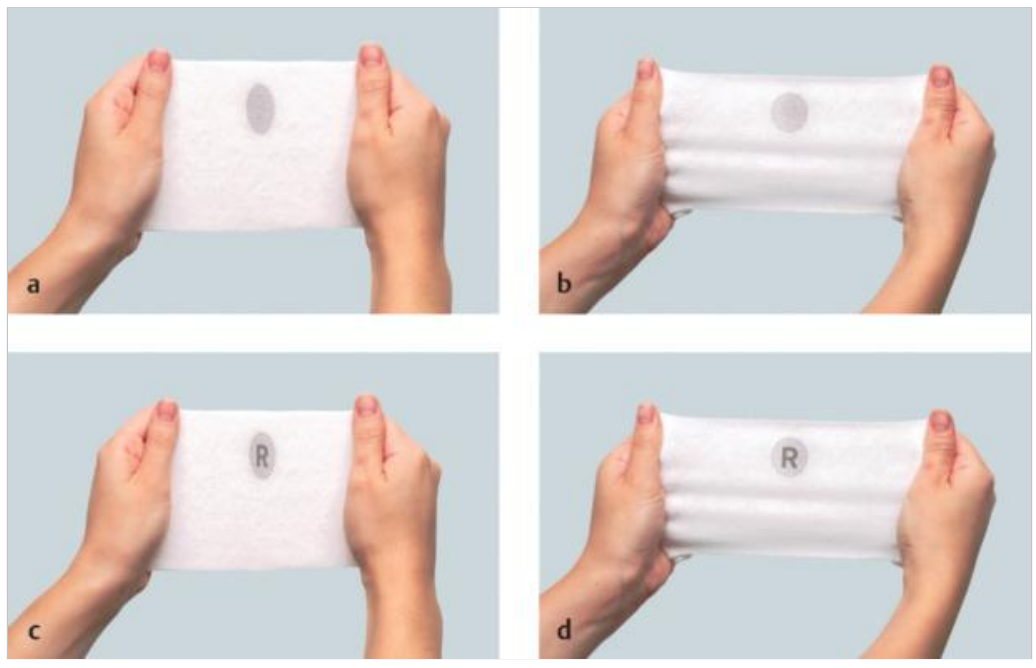

Abb. 5.10 Druckindikatoren der Kompressionsbinden.

(Quelle: Mit freundlicher Genehmigung der URGO GmbH)
a KTech ungedehnt.
b KTech gedehnt.
c KTech Lite ungedehnt.
d KTech Lite gedehnt.

\section{Applikationstechnik:}

- Anwendung nur einschließlich Unterschenkel

- Den Fuß im Sprunggelenk im rechten Winkel halten.

- Darauf achten, dass die wattierte Schicht (weiße polsternde Kurzzugbinde) in direktem Hautkontakt ist und die Markierung nach oben zeigt.

- Am Zehenansatz so anlegen (indem zwei Bindentouren zum Befestigen des Verbandes gewickelt werden), dass die Zehen 1-5 gerade eben nicht vom Bindenrand bedeckt sind.

- Den Knöchel in einer „Achter“-Tour umwickeln und dabei darauf achten, dass die Ferse vollständig bedeckt ist. 
- Der Fuß darf nicht mit vollständigem Zug umwickelt werden.

- Die Binde ab dem Knöchel spiralförmig um das Bein wickeln. Dabei nimmt der Anpressdruck aufgrund der anatomischen Beinform von distal nach proximal ab.

- Eine korrekte Überlappung ist erreicht, wenn der aufgedruckte Druckindikator durch die nächste Bahn teilweise bedeckt ist.

- Der Verband wird bis $2 \mathrm{~cm}$ unter der Kniekehle gewickelt, überschüssiges Material eventuell abgeschnitten und das Verbandsende mit einem Rollenpflaster fixiert.

- Die braune Binde (kohäsive Langzugbinde) wird in derselben Wickeltechnik wie die weiße Binde darüber angewendet. Dabei lässt man einen Rand der Polsterbinde an den Zehen und am Knie sichtbar, sodass sich nur die weiße Binde in direktem Hautkontakt befindet.

- Nach dem Anlegen des Verbands diesen flächenhaft leicht andrücken, um eine vollständige Selbsthaftung des Kompressionsverbandes sicherzustellen.

- Anwendung einschließlich Oberschenkel

- Gleichartige Applikation bei leicht gebeugtem Knie.

- Bei Bedarf für mehr Komfort zusätzlich die Kniekehle unter dem Verband abpolstern.

- Grundsätzlich ist auf die Vermeidung von Falten, Schnürfurchen, Schmerzen und Druckstellen zu achten.

- K2 und K2 Lite sollten Tag und Nacht bis zum nächsten Verbandwechsel getragen werden (entsprechend der ärztlichen Anordnung, maximal 7 Tage)

Wickeltechnik bei UrgoK2 und UrgoK2 Lite s. D Abb. 5.11. 


\section{Prophylaxe}
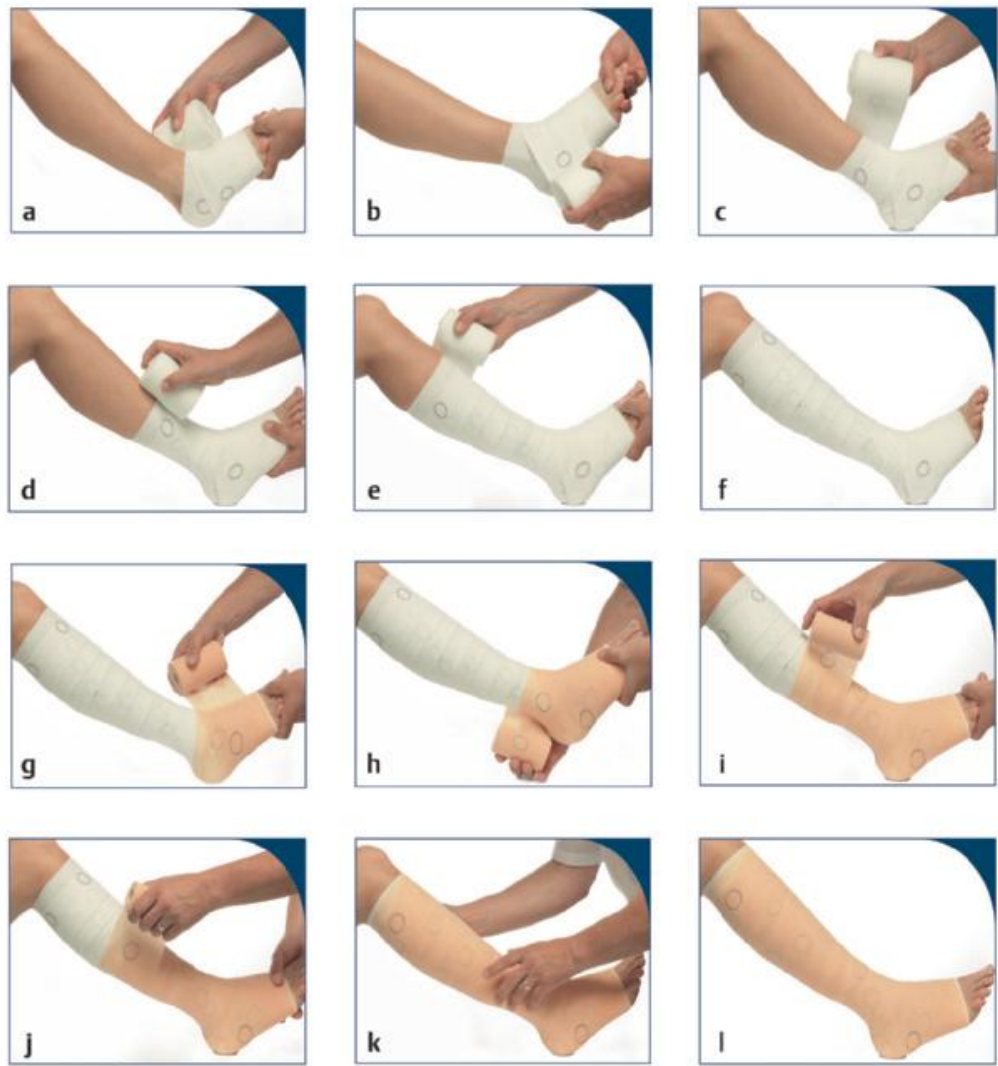

Abb. 5.11 Wickeltechnik bei UrgoK2 und UrgoK2 Lite. (Quelle: Mit freundlicher Genehmigung der URGO GmbH)

a KTech/KTech Lite am Zehenansatz anlegen, indem zwei Bindentouren zum Befestigen des Verbandes gewickelt werden. Darauf achten, dass die wattierte Schicht in direktem Hautkontakt ist.

b Den Knöchel mit einer Achtertour umwickeln.

c Die Ferse muss vollständig bedeckt sein. Der Fuß darf nicht mit vollständig ausgedehnter Markierung umwickelt werden.

d Die Binde ab dem Knöchel spiralförmig um das Bein nach oben wickeln, die Binde so dehnen, dass die Ellipsen einen Kreis bilden.

e Eine korrekte Überlappung ist erreicht, wenn der aufgedruckte Druckindikator an der Unterkante des Kreises bedeckt ist. 
Abb. 5.11 Fortsetzung; Wickeltechnik bei UrgoK2 und UrgoK2 Lite.

f Wickeln Sie den Verband bis $2 \mathrm{~cm}$ unter der Kniekehle. Schneiden Sie überschüssiges Material ab und fixieren Sie das Verbandende mit einem Rollenpflaster.

g Die zweite Lage KPress über KTeck/KTechLite wickeln und dabei dieselbe Wickeltechnik verwenden. Lassen Sie einen Rand der weißen Binde an de Zehen sichtbar, sodass sich nur KTech in direktem Hautkontakt befindet.

h Den Knöchel mit einer Achtertour umwickeln.

i Die Binde ab dem Knöchel spiralförmig um das Bein nach oben wickeln, die Binde so dehnen, dass die Ellipsen einen Kreis bilden.

j Eine korrekte Überlappung ist erreicht, wenn der aufgedruckte Druckindikator an der Unterkante des Kreises bedeckt ist.

k Wickeln Sie bis einen Finger breit unter KTech bzw. KTech Lite. Überschüssiges Material abschneiden. Nach dem Anlegen von KPress den Verband leicht andrücken, um eine vollständige Kohäsion sicherzustellen.

I Fertig angelegter Verband.

\section{Kontraindikationen}

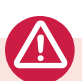

Relative Kontraindikationen: bei arterieller oder gemischt arterieller/venöser Erkrankung, bei fortgeschrittener diabetischer Mikroangiopathie (ärztlich angeordnete adaptierte Druckeinstellung möglich).

Absolute Kontraindikationen: fortgeschrittene arterielle Verschlusskrankheit, dekompensierte Herzinsuffizienz, Phlegmasia coerulea dolens und sonstige infektiöse oder septische Extremitätenprozesse. Bei Patienten mit Latexallergie kann z. B. alternativ UrgoK2 latexfrei angewendet werden.

\subsection{Therapie und Prävention mit Ligasano}

\section{Produkte}

\section{Gelistet in den Helios-Kliniken}

- Ligasano weiß steril $10 \times 10 \times 1 \mathrm{~cm}, 15 \times 10 \times 1 \mathrm{~cm}$

- Ligasano weiß steril Wundband $300 \times 2,5 \times 0,4 \mathrm{~cm}$

- Ligasano weiß steril Wundband mini $100 \times 1,5 \times 0,4 \mathrm{~cm}$

- Ligasano weiß steril $6 \times 2,5 \times 0,4 \mathrm{~cm}$

- Ligasano weiß steril Zehenschlauch groß 
- Ligasano weiß steril Zehenschlauch klein

- Ligasano weiß steril Zylinder

- Ligasano weiß steril Hallux-valgus-Verband

- Ligasano grün unsteril $55 \times 45 \times 2 \mathrm{~cm}$

- Ligasano grün steril $15 \times 10 \times 2 \mathrm{~cm}$

- Ligasano Fersenschuh, passend für alle Fußgrößen

- Ligasano Wundputzer medium steril $5 \times 5 \times 2 \mathrm{~cm}$ orange

- Ligasano weiß steril Zehenschlauch/Zehenkappe $14 \times 10 \times 0,5 \mathrm{~cm}$

- Ligasano weiß unsteril Spenderbox $200 \times 11 \times 1 \mathrm{~cm}$

- Ligasano weiß unsteril goße Platten $59 \times 49 \times 1 \mathrm{~cm}$

- Ligasano weiß unsteril $29 \times 12 \times 1 \mathrm{~cm}$ Innenhandverband

- Ligasano grün unsteril Spenderbox $200 \times 11 \times 1 \mathrm{~cm}$

- Ligasano weiß unsteril Ring $\varnothing 15 \times 8 / 5 \mathrm{~cm}$

- Ligasano orange unsteril Omega Polster 5-fach verstellbar + 5 HautkontaktPolster $15 \times 10 \times 1 \mathrm{~cm}$ Ligasano weiß unsteril

- Ligasano weiß (steril) Ø $20 \times 1 \mathrm{~cm}$.

- Ligasano grün unsteril $49 \times 49 \times 9 / 1 \mathrm{~cm}$ (Keil)

- Ligasano weiß unsteril Binden $300 \times 5 \times 0,3 \mathrm{~cm}, 300 \times 10 \times 0,3 \mathrm{~cm}$

- Ligasano weiß unsteril Zehenschlauch 6x3,3/2,5/1,5 x0,5 cm

- Ligasano weiß unsteril $15 \times 10 \times 1 \mathrm{~cm}$

Alle (LIG) ( $\triangleright$ Abb. 5.12).

\section{Vergleichbares Produkt}

Darco Heelift Fersenentlastungsschuh.

Tab. 5.2 Produkteigenschaften.

\section{Eigenschaften der oben genannten Produkte:}

\begin{tabular}{|c|c|c|}
\hline $0-0$ & $s$ & I:: \\
\hline
\end{tabular}



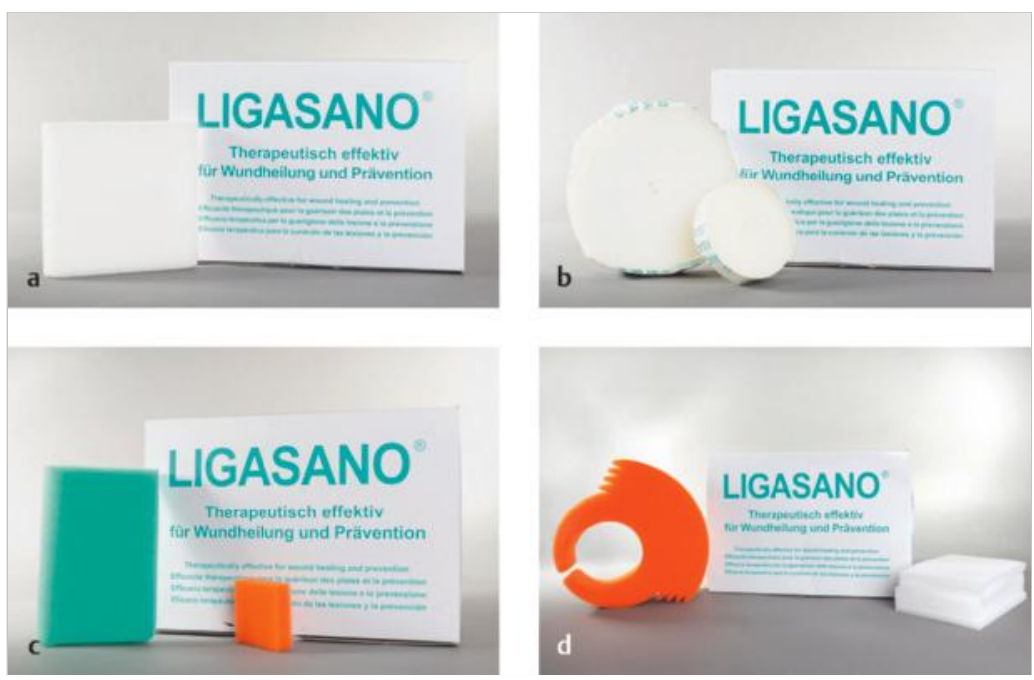

Abb. 5.12 Ligasano.

a Ligasano weiß.

b Ligasano weiß, Tamponade.

c Ligasano grün und Wundputzer.

d Ligasano orange unsteril Omega Polster 5-fach verstellbar + 5 Hautkontakt-Polster.

\subsubsection{Besondere Eigenschaften}

- Schnell nachlassende Druckspannung; reduziert den Druck auf das Gewebe und den Gegendruck.

- Der mechanische Reiz fördert die Durchblutung.

- Besitzt eine hohe Durchlässigkeit für Wasserdampf, ist atmungsaktiv und gebremst luftdurchlässig.

- Die Druckspannung ist bei grün wenig nachlassend und bei weiß schnell nachlassend (ca. $40 \%$ nach 20 Minuten).

- Ligasano grün ist je nach Belastung bis zu mehreren Wochen einsetzbar. Dieses Produkt ist patientenbezogen waschbar. 


\section{Prophylaxe}

\subsubsection{Indikation}

- Dekubitusprophylaxe (Druckverteilung, Druckentlastung, Mikroklima, Scherkräfte mindern)

- Intertrigobehandlung und -prophylaxe, Begleitbehandlung bei Mykosen $\rightarrow$ reguliert die Hautfeuchte

- Polsterung und Schienung von Kanülen, Kathetern, Drainagen und Ableitungen.

\section{Kontraindikationen}

Grüner und orangefarbener Schaum darf nicht direkt auf die Haut aufgelegt werden. Eine mechanische Reinigung mit diesen Schäumen ist möglich.

Vorteile:

- allergieneutral

- vielseitig einsetzbar

- einfache Anwendung.

\subsubsection{Anwendungsweise}

- Ligasano weiß und grün können zugeschnitten werden.

- Eine Fixierung ist oftmals notwendig.

\section{Merke}

Eine Anwendungsschulung wird dringend empfohlen!

Anwendungsbeispiele s. > Abb. 5.13, > Abb. 5.14, > Abb. 5.15.

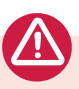




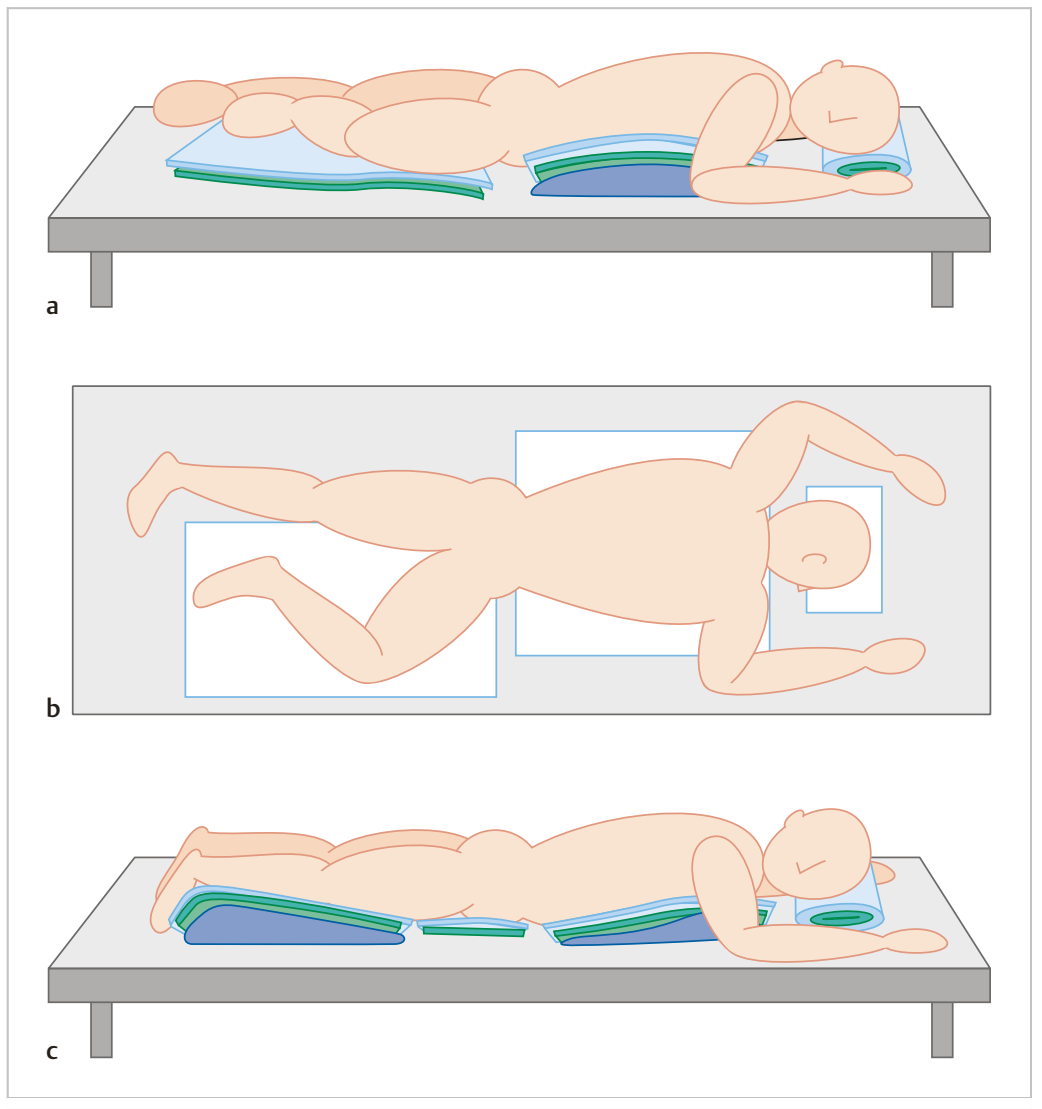

Abb. 5.13 Anwendungsbeispiel Bauchlage.

a Liegendansicht seitlich $135^{\circ}$.

b Liegendansicht von oben $135^{\circ}$.

c Bauchlage $180^{\circ}$. 


\section{Prophylaxe}

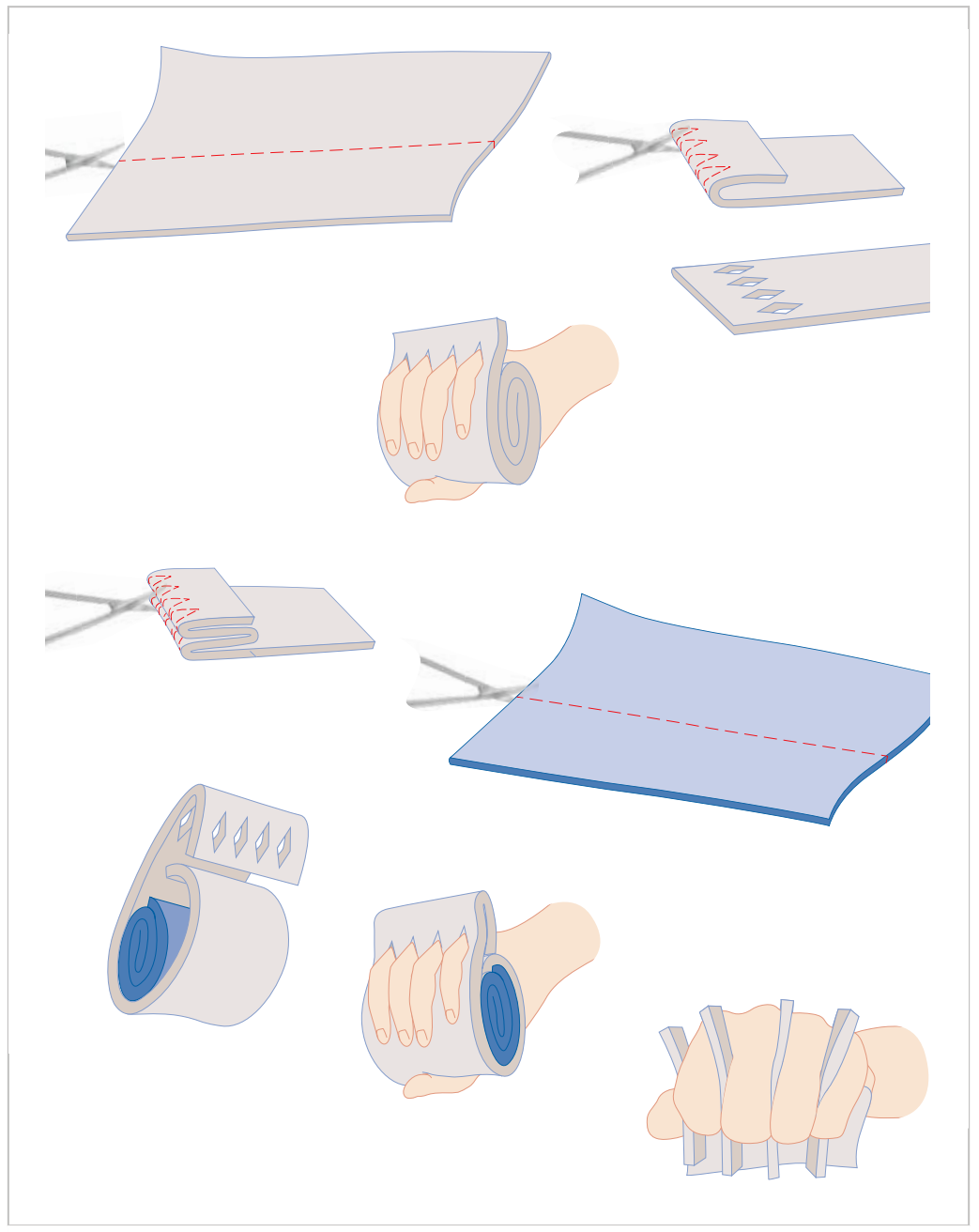

Abb. 5.14 Anwendungsbeispiel Fingerpolsterung. 


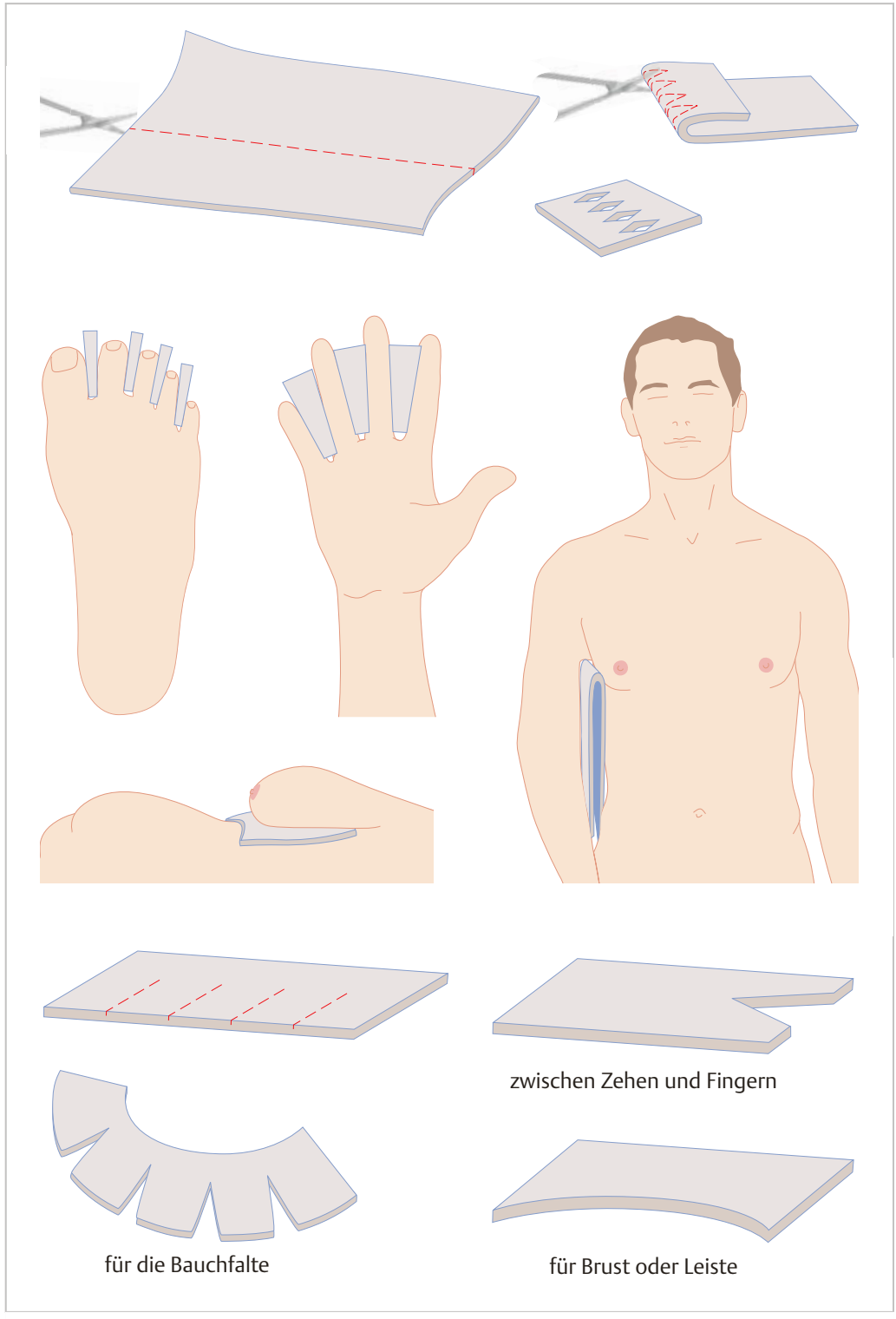

Abb. 5.15 Anwendungsbeispiel Intertrigoprophylaxe. 


\subsection{Ernährung und Wunde}

Der Ernährungszustand des Patienten spielt eine wichtige Rolle bei der Wundheilung. So kann ein ungewollter Gewichtsverlust das Risiko für einen Dekubitus erhöhen.

Ein Ernährungsdefizit kann die Entstehung chronischer Wunden fördern und verschlechtert die Wundheilung. Körperliche Beeinträchtigungen, Störungen der Nahrungsaufnahme z. B. durch Appetitmangel oder Schluckbeschwerden, aber auch belastende Situationen oder Schmerzen können Patienten davon abhalten, sich ausgewogen zu ernähren.

Eine Beratung und Unterstützung durch ein Ernährungsteam ist sinnvoll.

Nach Benedikt und Weitgasser sollte Ernährung als Teil der gesamtmedizinischen Therapie chronischer Wunden betrachtet werden [1]. Ein frühzeitig eingesetztes Behandlungskonzept für Patienten mit Wundheilungsstörungen kann einerseits Läsionen verhindern und andererseits eine raschere Abheilung bestehender Wunden unterstützen.

Besonders Dekubitalulzera haben oft einen langwierigen und komplizierten Verlauf. Eine optimale Ernährung kann eine schnellere Wundheilung bewirken.

Um den Ernährungszustand eines Patienten erfassen zu können, gibt es eine Reihe von Hilfsmitteln und Messmethoden:

- Anamnese (Diäten, Essverhalten, Gewichtsabnahme etc.)

- klinische Untersuchung (Schluckstörungen, Depressionen, Übelkeit, Demenz, Medikamente etc.)

- Messmethoden (Größe und Gewicht, BMI, Hautfaltenmessung)

- Laborwerte können ebenfalls Aufschluss über den Ernährungszustand geben (Blutzucker, Cholesterin, Albumin, Hämoglobin, Gesamteiweiß, Spurenelemente wie Zink etc.).

\subsubsection{Handlungsempfehlung der Helios-Fachgruppe Pflege zum Ernährungsmanagement}

1. Definition „Mangelernährung“: „Ein anhaltendes Defizit an Energie und/ oder Nährstoffen im Sinne einer negativen Bilanz zwischen Aufnahme und Bedarf mit Konsequenzen und Einbußen für Ernährungszustand, physiologische Funktionen und Gesundheitszustand“ [7].

2. Ziel: Ziel ist die bedarfsgerechte, bedürfnisorientierte und angemessene orale Versorgung und Unterstützung von stationären erwachsenen Patienten während ihres stationären Aufenthalts. 


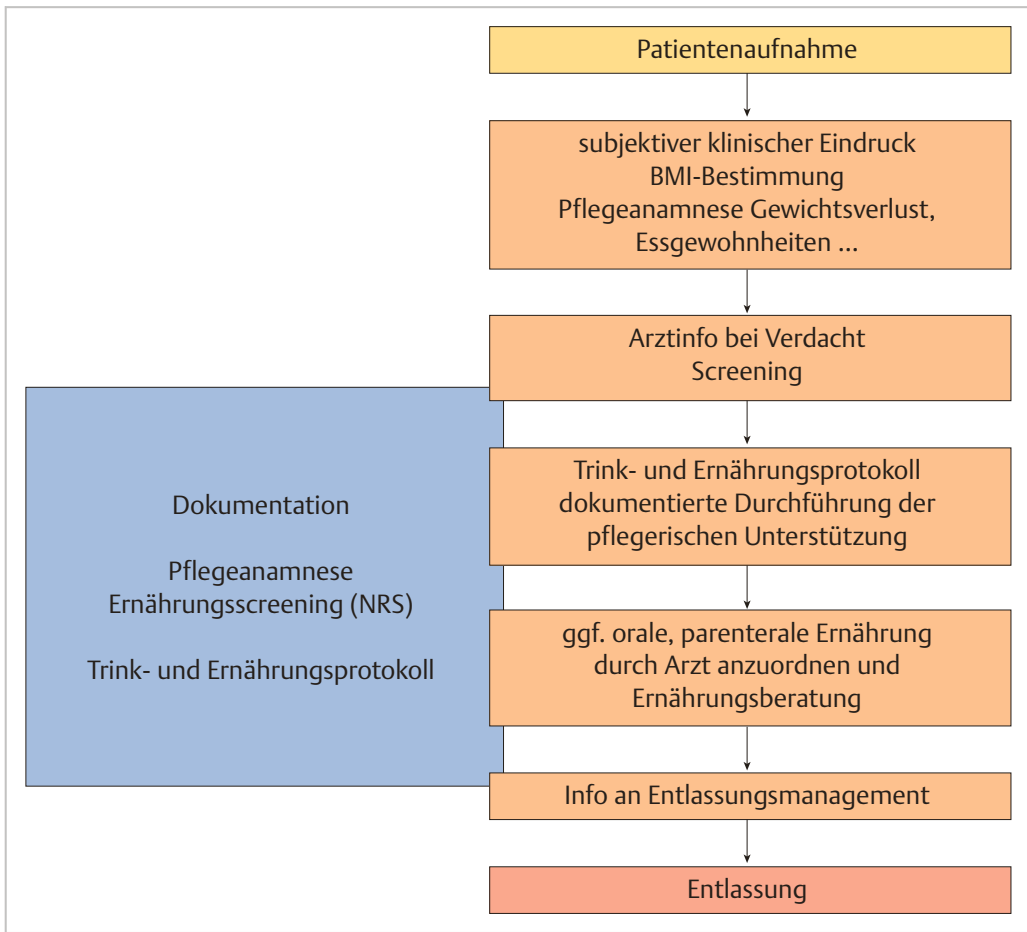

Abb.5.16 Algorithmus Ernährungsmanagement.

3. Durchführung: s. \Abb. 5.16.

\section{Dokumentation}

Hausspezifische Dokumentationsrichtlinien beachten:

- BMI (Body-Mass-Index) bei Aufnahme/Veränderung/Entlassung erheben

- Pflegeanamnese (Gewichtsabnahme, Essgewohnheiten, Appetit)

- Ernährungsscreening (Nutritional Risk Screening)

- Trink- und Ernährungsprotokoll.

\section{Evaluation}

- Gewicht, BMI

- veränderte Gewohnheiten und Vorlieben

- Unterstützungsbedarf bei der Nahrungsaufnahme. 


\section{Prophylaxe}

\section{Mitgeltende Dokumente}

6.1 Expertenstandard Ernährungsmanagement zur Sicherstellung und Förderung der oralen Ernährung in der Pflege [6]

\section{Besonders wichtig:}

- Risikoerfassung und Pflegeanamnese innerhalb von 24 Stunden nach Aufnahme

- Unterstützung des Patienten bei der Nahrungsaufnahme

- Informationsweitergabe an Entlassungsmanagement.

\section{Literaturhinweise}

- Schmidt, Simone: Expertenstandards in der Pflege. Eine Gebrauchsanleitung. 4. aktualisierte Auflage. Berlin, Heidelberg Springer 2020

- Erhebungsbögen zur Erfassung von Mangelernährung z. B. Screening Mangelernährung (SEM; • Tab. 5.3).

\subsubsection{Therapie der Mangelernährung}

Die Therapien der Mangelernährung sind - ebenso wie deren Ursachen - sehr vielfältig. Sie reichen von alleiniger Substitution von Vitaminen bis zu komplizierten hochkalorischen Infusionsprogrammen. Die Optimierung der Wundheilung (zumindest großer Wundflächen) erfordert die Therapie der Mangelernährung.

\section{Merke}

Bei jedem Patienten ist auf Zeichen der Mangelernährung zu achten! 
Tab. 5.3 Screening auf Mangelernährung im Krankenhaus.

\begin{tabular}{|l|l|}
\hline Nutritional Risk Screening (NRS 2002)* & \\
\hline Vorscreening & Ja/Nein \\
\hline Ist der Body Mass Index $<20,5 \mathrm{~kg} / \mathrm{m}^{2}$ ? & Ja/Nein \\
\hline Hat der Patient in den vergangenen 3 Monaten an Gewicht verloren? & Ja/Nein \\
\hline War die Nahrungszufuhr in der vergangenen Woche vermindert? & Ja/Nein \\
\hline Ist der Patient schwer erkrankt? (z. B. Intensivtherapie) &
\end{tabular}

- Wird eine dieser Fragen mit „Ja“ beantwortet, wird mit dem Hauptscreening fortgefahren.

- Werden alle Fragen mit „Nein“ beantwortet, wird der Patient wöchentlich neu gescreent.

- Wenn für den Patienten z. B. eine große Operation geplant ist, sollte ein präventiver Ernährungsplan verfolgt werden, um dem assoziierten Risiko vorzubeugen.

\section{Hauptscreening}

\begin{tabular}{l} 
Störung des Ernährungszustands \\
\hline Keine \\
\hline Mild: Gewichtsverlust $>5 \% / 3$ Mon. \\
oder Nahrungszufuhr $<50-75 \%$ des \\
Bedarfs in der vergangenen Woche
\end{tabular}

Mäßig: Gewichtsverlust > 5\%/2 Mon. 2 oder BMI $18,5-20,5 \mathrm{~kg} / \mathrm{m}^{2}$ und reduzierter Allgemeinzustand (AZ) oder Nahrungszufuhr $20-60 \%$ des Bedarfs in der vergangenen Woche

Schwer: Gewichtsverlust>5\%/

\begin{tabular}{|l|l|}
\hline Punkte & Krankheitsschwere \\
\hline $\mathbf{0}$ & Keine \\
\hline $\mathbf{1}$ & Mild: $\mathrm{z}$. B. Schenkelhals- \\
& fraktur, chronische Erkran- \\
& kungen besonders mit \\
& Komplikationen: Leberzir- \\
& rhose, chronisch obstruk- \\
tive Lungenerkrankung, \\
chronische Hämodialyse, \\
Diabetes, Krebsleiden
\end{tabular}
Punkte 0

Mäßig: z. B. große Bauchchirurgie, Schlaganfall, schwere Pneumonie, hämatologische Krebserkrankung

1 Mon. (> 15\%/3 Mon.) oder BMI $18,5-20,5 \mathrm{~kg} / \mathrm{m}^{2}$ und reduzierter Allgemeinzustand (AZ) oder Nahrungszufuhr $0-25 \%$ des Bedarfs in der vergangenen Woche

Schwer: z. B. Kopfverletzung, Knochenmarktransplantation, intensivpflichtige Patienten (APACHE-II $>10)$

+1 Punkt, wenn Alter $\geq 70$ Jahre

\section{Bewertung}

$\geq 3$ Punkte: Ernährungsrisiko liegt vor, Erstellung eines Ernährungsplans.

$\leq 3$ Punkte: Wöchentlich wiederholtes Screening. Wenn für den Patienten z. B. eine große Operation geplant ist, sollte ein präventiver Ernährungsplan verfolgt werden, um das assoziierte Risiko zu vermeiden.

* Nach Kondrup J et al., Clinical Nutrition 2003; 22:415-421. Empfohlen von der Europäischen Gesellschaft für Klinische Ernährung und Stoffwechsel (ESPEN). 


\subsection{Edukation von Patienten und Angehörigen}

Die Patientenedukation gehört mit zu der Hauptaufgabe bei der praktischen Tätigkeit aller Beteiligten in der Patientenversorgung.

Meist erfolgt sie direkt im Gespräch mit dem Patienten während einer Pflegehandlung oder kann mittels Schulungen, wie z.B. Wundberatung, Ernährungsberatung geschehen.

Wichtig ist, Familienmitglieder und betreuende Pflegepersonen mit in den Prozess aufzunehmen.

Definition: Die Patientenedukation geht der Frage nach, welches Wissen und Können Menschen benötigen, um im Alltag mit der Wunde umzugehen. Sie beinhaltet alle Formen der Wissensvermittlung wie strukturierte Schulung, Anleitung, Information und Aufklärung. Sie beinhaltet aber auch die professionelle Beratung zu Fragen von Patienten und Angehörigen.

Der Aspekt „Beratung und Schulung“ basiert auf Übersichtsarbeiten, Primärstudien sowie Empfehlungen aus Leitlinien.

Das Deutsche Netzwerk für Qualitätsentwicklung in der Pflege (DQNP) beschreibt Struktur-Prozess-Ergebniskriterien wie folgt:

- Strukturkriterien: Die Pflegefachkraft verfügt über aktuelles Wissen und Kompetenz zu Information, Beratung, Schulung und Anleitung zum gesundheitsbezogenen Selbstmanagement. Die Einrichtung stellt zielgruppenspezifische Materialien für Information, Beratung, Schulung und Anleitung zur Verfügung.

- Prozesskriterien: Die Pflegefachkraft schult zu Wundursachen und fördert die Fähigkeiten des Patienten/Bewohners und seiner Angehörigen zur Wundversorgung sowie zum Umgang mit wund- und therapiebedingten Einschränkungen durch Maßnahmen der Patientenedukation. Sie unterstützt die Kontaktaufnahme zu anderen Berufs-, Selbsthilfe- oder weiteren Gesundheitsgruppen.

- Ergebniskriterien: Der Patient/Bewohner und seine Angehörigen kennen die Ursache der Wunde sowie die Bedeutung der vereinbarten Maßnahmen und sind über weitere Unterstützungsmöglichkeiten informiert. Ihr gesundheitsbezogenes Selbstmanagement ist entsprechend ihrer individuellen Möglichkeiten gefördert. 